\title{
Upper and lower bounds on the set of recoverable strains and on effective energies in cubic-to-monoclinic martensitic phase transformations
}

\author{
Anja Schlömerkemper ${ }^{1}$, a , Isaac V. Chenchiah ${ }^{2}$, Rainer Fechte-Heinen ${ }^{3}$, and Daniel Wachsmuth ${ }^{1}$ \\ ${ }^{1}$ University of Würzburg, Institute for Mathematics, Emil-Fischer-Str. 40, 97074 Würzburg, Germany \\ ${ }^{2}$ University of Bristol, School of Mathematics, University Walk, Bristol, BS8 1TW, UK \\ ${ }^{3}$ Ruhr-University Bochum, Institute for Mechanics, 44780 Bochum, Germany
}

\begin{abstract}
A major open problem in the mathematical analysis of martensitic phase transformations is the derivation of explicit formulae for the set of recoverable strains and for the relaxed energy of the system. These are governed by the mathematical notion of quasiconvexity. Here we focus on bounds on these quasiconvex hulls and envelopes in the setting of geometrically-linear elasticity. Firstly, we will present mathematical results on triples of transformation strains. This yields further insight into the quasiconvex hull of the twelve transformation strains in cubic-to-monoclinic phase transformations. Secondly, we consider bounds on the energy of such materials based on the so-called energy of mixing thus obtaining a lamination upper bound on the quasiconvex envelope of the energy. Here we present a new algorithm that yields improved upper bounds and allows us to relate numerical results for the lamination upper bound on the energy with theoretical inner bounds on the quasiconvex hull of triples of transformation strains.
\end{abstract}

\section{Introduction}

In this article we study martensitic phase transformations in the context of shape memory alloys (SMA), which have a large variety of technological applications, e.g., in medical or satellite technology. SMAs are a class of materials which show very flexible behaviour known as pseudoelasticity (stress based) and shape-memory effect (temperature based). This relies on a spontaneous diffusion-less solid-solid phase transformation from austenite to martensite and vice-versa. A key feature is the formation of finescale microstructures in the martensitic phase composed of different martensitic variants.

Though these materials are very flexible, there is a limit after which the material deforms plastically. This article is concerned with the question of characterizing this limit. Phrased differently, we aim to characterize all strains that allow recovery of the original shape of the material upon heating above a critical temperature. We follow the variational approach proposed by Ball and James [1], see [2] for an introduction. The mathematical notion related to the addressed question is that of quasiconvexity (in the sense of Morrey). The objects we seek are the so-called quasiconvex hull of the set of (phase) transformation strains and the quasiconvex envelope of the related free energy density. Unfortunately these are extremely difficult to evaluate. Quasiconvex hulls are known only in some special cases. One therefore considers inner and outer bounds on the quasiconvex hull such as the poly-

\footnotetext{
ae-mail: anja.schloemerkemper@mathematik.uni-wuerzburg.de
}

convex hull, the rank-one convex hull and the lamination convex hull, respectively, see, e.g., [3, 4] for details.

We work in the setting of geometrically-linear elasticity and focus on mono-crystalline materials. We assume that each variant obeys a linear-elastic material law from which we obtain the energy

$$
W_{j}(\boldsymbol{e})=\frac{1}{2}\left(\boldsymbol{e}-\boldsymbol{e}^{(j)}\right): \mathbb{C}:\left(\boldsymbol{e}-\boldsymbol{e}^{(j)}\right),
$$

where $e^{(j)}$ is the transformation strain and $\mathbb{C}$ is the (variantindependent) elastic modulus. This yields the microscopic energy density

$$
W(\boldsymbol{e})=\min _{j=1, \ldots, n} W_{j}(\boldsymbol{e})
$$

where $n$ is the number of variants. The overall effective macroscopic energy is then given by the relaxation of the microscopic energy, which corresponds to calculating the quasiconvex envelope, $Q W(\boldsymbol{e}, \boldsymbol{c})$, corresponding to the vector of variant fractions

$$
c \in \Delta_{n}:=\left\{c \in \mathbb{R}^{n}: c_{i} \geq 0, i=1, \ldots, n, \sum_{i=1}^{n} c_{i}=1\right\} .
$$

If the individual transformation strains are pairwise compatible (see Def. 1 below), it is known [5] that their quasiconvex hull/envelope and convex hull/envelope coincide. This applies to the cases of cubic-to-tetragonal, cubic-to-trigonal and cubic-to-orthorhombic phase transformations but it does not apply to cubic-to-monoclinic phase transformations, which however are of great interest, e.g., in NiTi, which has a wide industrial application.

This is an Open Access article distributed under the terms of the Creative Commons Attribution License 4.0, which permits unrestricted use distribution, and reproduction in any medium, provided the original work is properly cited. 
Table 1. The transformation strains of the 12 variants of monoclinic-I martensite, cf. eg., [6, Table 1]. In the computations we choose the material parameters for NiTi: $\alpha=0.0243, \beta=-0.0437, \delta=0.0580$ and $\varepsilon=0.0427$, cf. [6].

\begin{tabular}{l|l||l|l||l|l}
$\mathrm{i}$ & $\boldsymbol{e}^{(i)}$ & $\mathrm{i}$ & $\boldsymbol{e}^{(i)}$ & $\mathrm{i}$ & $\boldsymbol{e}^{(i)}$ \\
\hline 1 & $\left(\begin{array}{ccc}\alpha & \delta & \varepsilon \\
\delta & \alpha & \varepsilon \\
\varepsilon & \varepsilon & \beta\end{array}\right)$ & 5 & $\left(\begin{array}{ccc}\alpha & \varepsilon & \delta \\
\varepsilon & \beta & \varepsilon \\
\delta & \varepsilon & \alpha\end{array}\right)$ & 9 & $\left(\begin{array}{ccc}\beta & \varepsilon & \varepsilon \\
\varepsilon & \alpha & \delta \\
\varepsilon & \delta & \alpha\end{array}\right)$ \\
\hline 2 & $\left(\begin{array}{ccc}\alpha & \delta & -\varepsilon \\
\delta & \alpha & -\varepsilon \\
-\varepsilon & -\varepsilon & \beta\end{array}\right)$ & 6 & $\left(\begin{array}{ccc}\alpha & -\varepsilon & \delta \\
-\varepsilon & \beta & -\varepsilon \\
\delta & -\varepsilon & \alpha\end{array}\right)$ & 10 & $\left(\begin{array}{ccc}\beta & -\varepsilon & -\varepsilon \\
-\varepsilon & \alpha & \delta \\
-\varepsilon & \delta & \alpha\end{array}\right)$ \\
\hline 3 & $\left(\begin{array}{ccc}\alpha & -\delta & -\varepsilon \\
-\delta & \alpha & \varepsilon \\
-\varepsilon & \varepsilon & \beta\end{array}\right)$ & 7 & $\left(\begin{array}{ccc}\alpha & -\varepsilon & -\delta \\
-\varepsilon & \beta & \varepsilon \\
-\delta & \varepsilon & \alpha\end{array}\right)$ & 11 & $\left(\begin{array}{ccc}\beta & -\varepsilon & \varepsilon \\
-\varepsilon & \alpha & -\delta \\
\varepsilon & -\delta & \alpha\end{array}\right)$ \\
\hline 4 & $\left(\begin{array}{ccc}\alpha & -\delta & \varepsilon \\
-\delta & \alpha & -\varepsilon \\
\varepsilon & -\varepsilon & \beta\end{array}\right)$ & 8 & $\left(\begin{array}{ccc}\alpha & \varepsilon & -\delta \\
\varepsilon & \beta & -\varepsilon \\
-\delta & -\varepsilon & \alpha\end{array}\right)$ & 12 & $\left(\begin{array}{ccc}\beta & \varepsilon & -\varepsilon \\
\varepsilon & \alpha & -\delta \\
-\varepsilon & -\delta & \alpha\end{array}\right)$
\end{tabular}

Thus, here we consider cubic-to-monoclinic (specifically monoclinic-I) phase transformations. These are described by the 12 transformation strains in $\mathcal{E}:=$ $\left\{\boldsymbol{e}^{(1)}, \ldots, \boldsymbol{e}^{(12)}\right\} \subset \mathcal{S}_{c}^{3 \times 3}$, where $\mathcal{S}_{c}^{3 \times 3}$ denotes real symmetric $3 \times 3$-matrices $\mathcal{S}^{3 \times 3}$ with constant trace, cf. Table 1 . We introduce a new algorithm to compute an upper bound on the quasiconvex envelope of the free energy density. This is based on the so-called energy of mixing and sequential lamination, described in $\S 3$. We discover that for so-called $\mathrm{T}_{3}$-configurations, cf. $\S 2$, the (almost) zero set of this bound is very close to the corresponding inner bound on the quasiconvex hull of the related strains. This inner bound is obtained by considering the compatible (ie., symmetrized rank-one convex) hull of the three strains involved. These can be classified as follows:

Case 1: Pairwise incompatible which form $\mathrm{T}_{3} \mathrm{~s}$, e.g., $\left\{\boldsymbol{e}^{(1)}, \boldsymbol{e}^{(6)}, \boldsymbol{e}^{(12)}\right\}$,

Case 2: Two incompatible pairs and one compatible pair, e.g., $\left\{\boldsymbol{e}^{(1)}, \boldsymbol{e}^{(7)}, \boldsymbol{e}^{(12)}\right\}$ and $\left\{\boldsymbol{e}^{(1)}, \boldsymbol{e}^{(6)}, \boldsymbol{e}^{(8)}\right\}$,

Case 3: Two compatible pairs and one incompatible pair with no compatibility between the compatible lines, e.g., $\left\{\boldsymbol{e}^{(1)}, \boldsymbol{e}^{(7)}, \boldsymbol{e}^{(10)}\right\}$,

Case 4: Two compatible pairs and one incompatible pair with some compatibility between the compatible lines, e.g., $\left\{\boldsymbol{e}^{(1)}, \boldsymbol{e}^{(3)}, \boldsymbol{e}^{(12)}\right\}$ or $\left\{\boldsymbol{e}^{(1)}, \boldsymbol{e}^{(2)}, \boldsymbol{e}^{(7)}\right\}$,

Case 5: Pairwise compatible, e.g., $\left\{\boldsymbol{e}^{(1)}, \boldsymbol{e}^{(2)}, \boldsymbol{e}^{(3)}\right\}$.

We study the corresponding compatible hulls theoretically in $\$ 2$ and investigate the envelope of the corresponding energy numerically in $\S 4$, this is motived by [7] and [8], respectively. A comparison of the results yields a striking correspondence.

\section{Stress-free situation}

In the stress-free situation we are interested in the zero, i.e., minimal, energy states of the system which are given by the quasiconvex hull of $\mathcal{E}$. In order to obtain some further insight into this task, we follow ideas of $[7,8]$ and consider inner/upper and outer/lower bounds on the quasiconvex hull/envelope of triples of these 12 strains. It turns out that these depend on compatibility properties of the strains:

Definition 1. Let $\boldsymbol{e}_{1}, \boldsymbol{e}_{2} \in \mathcal{S}_{c}^{3 \times 3}$. Then $\boldsymbol{e}_{1}$ and $\boldsymbol{e}_{2}$ are compatible (or $\boldsymbol{e}_{1}$ and $\boldsymbol{e}_{2}$ are symmetrized rank-one connected), $\boldsymbol{e}_{1} \| \mid \boldsymbol{e}_{2}$, if there exist $\boldsymbol{a}, \boldsymbol{b} \in \mathbb{R}^{3}$ such that $\boldsymbol{e}_{1}-\boldsymbol{e}_{2}=$ $\frac{1}{2}(\boldsymbol{a} \otimes \boldsymbol{b}+\boldsymbol{b} \otimes \boldsymbol{a})$, where $\otimes$ denotes the dyadic product. Otherwise they are said to be incompatible, $\boldsymbol{e}_{1} \mid \boldsymbol{\|} \boldsymbol{e}_{2}$.

A simple characterization of compatibility in $\mathcal{S}_{c}^{3 \times 3}$ obtained from [9, Lemma 4.1], cf. [7, Lemma 2.2] is:

$$
\boldsymbol{e}_{1} \| \boldsymbol{e}_{2} \quad \text { iff } \quad \operatorname{det}\left(\boldsymbol{e}_{1}-\boldsymbol{e}_{2}\right)=0 .
$$

This allows to easily determine specific examples for the five cases above, see [7] for details and further examples.

The exact definition of the quasiconvex hull $Q(\mathcal{E})$ of $\mathcal{E}$ is not relevant here. We therefore refer to [7] and references therein for the definition and for further definitions and properties stated below. Crucial for the following considerations are the bounds

$$
\mathcal{L}(\mathcal{E}) \subseteq \mathcal{R}(\mathcal{E}) \subseteq Q(\mathcal{E}) \subseteq \mathcal{C}(\mathcal{E}),
$$

where $C(\mathcal{E})$ denotes the convex hull and $\mathcal{L}(\mathcal{E})$ the lamination convex hull of $\mathcal{E}$. However we need the definition of the symmetrized rank-one convex hull, $\mathcal{R}(\mathcal{E})$, which is based on the notion of symmetrized rank-one convex functions:

Definition 2. A function $f: \mathcal{S}^{3 \times 3} \rightarrow \mathbb{R}$ is symmetrized rank-one convex if for all $\lambda \in[0,1]$ and for all $\boldsymbol{e}_{1}, \boldsymbol{e}_{2} \in$ $\mathcal{S}^{3 \times 3}$ with $\boldsymbol{e}_{1}|| \boldsymbol{e}_{2}$,

$$
f\left(\lambda \boldsymbol{e}_{1}+(1-\lambda) \boldsymbol{e}_{2}\right) \leqslant \lambda f\left(\boldsymbol{e}_{1}\right)+(1-\lambda) f\left(\boldsymbol{e}_{2}\right) .
$$

Definition 3. Let $E \subset \mathcal{S}^{3 \times 3}$ be compact. Then the symmetrized rank-one convex hull of $E$ is

$$
\begin{aligned}
\mathcal{R}(E) & :=\left\{\boldsymbol{e} \in \mathcal{S}^{3 \times 3} \mid f(\boldsymbol{e})=0 \quad \forall f: \mathcal{S}^{3 \times 3} \rightarrow[0, \infty)\right. \\
& \text { symmetrized rank-one convex with } f(E)=\{0\}\}
\end{aligned}
$$

We begin our analysis with a well-known result:

Lemma $1([5, \S 3.4 .1])$. Let $E \subset \mathcal{S}^{3 \times 3}$ be finite and let all elements of $E$ be pairwise compatible. Then $\mathcal{L}(E)=C(E)$.

Moreover, for all $\boldsymbol{e}_{1}, \boldsymbol{e}_{2} \in \mathcal{S}_{c}^{3 \times 3}$

$$
\mathcal{R}\left(\left\{\boldsymbol{e}_{1}, \boldsymbol{e}_{2}\right\}\right)= \begin{cases}{\left[\boldsymbol{e}_{1}, \boldsymbol{e}_{2}\right]} & \text { if } \boldsymbol{e}_{1} \| \mid \boldsymbol{e}_{2}, \\ \left\{\boldsymbol{e}_{1}, \boldsymbol{e}_{2}\right\} & \text { else, }\end{cases}
$$

where $\left[\boldsymbol{e}_{1}, \boldsymbol{e}_{2}\right]:=\left\{\lambda \boldsymbol{e}_{1}+(1-\lambda) \boldsymbol{e}_{2} \mid \lambda \in[0,1]\right\}$. Further, if $E$ consists of three pairwise compatible strains as in Case 5, then $\mathcal{R}(E)=C(E)$ is the triangle with these strains as extreme points.

Next we will recall some results related to Case 1:

Definition 4. Three points $\boldsymbol{e}_{1}, \boldsymbol{e}_{2}, \boldsymbol{e}_{3} \in \mathcal{S}_{c}^{3 \times 3}$ form a $\mathrm{T}_{3}$ if

\section{They are pairwise incompatible, and}


2. There exist $\boldsymbol{e}_{1,1} \in\left(\boldsymbol{e}_{2}, \boldsymbol{e}_{3}\right), \boldsymbol{e}_{2,2} \in\left(\boldsymbol{e}_{3}, \boldsymbol{e}_{1}\right), \boldsymbol{e}_{3,3} \in$ $\left(\boldsymbol{e}_{1}, \boldsymbol{e}_{2}\right)$ such that $\boldsymbol{e}_{i, i} \| \mid \boldsymbol{e}_{i}, i=1,2,3$.

The following $\mathrm{T}_{3}$-lemma [7, Lemma 4.3] yields an easy characterization of $\mathrm{T}_{3} \mathrm{~s}$ :

Lemma 2. Three points $\boldsymbol{e}_{1}, \boldsymbol{e}_{2}, \boldsymbol{e}_{3} \in \mathcal{S}_{c}^{3 \times 3}$ form a $T_{3}$ iff $\operatorname{sign} \operatorname{det}\left(\boldsymbol{e}_{1}-\boldsymbol{e}_{2}\right)=\operatorname{sign} \operatorname{det}\left(\boldsymbol{e}_{2}-\boldsymbol{e}_{3}\right)=\operatorname{sign} \operatorname{det}\left(\boldsymbol{e}_{3}-\boldsymbol{e}_{1}\right) \neq 0$.

It turns out that all triples of pairwise incompatible strains in $\mathcal{E}$ are $\mathrm{T}_{3} \mathrm{~s}$, see [7, $\S 3$ and $\left.\S 7\right]$. For $\mathrm{T}_{3} \mathrm{~s}$ it is known (cf., e.g., $[10, \S 3])$ that

$$
\begin{array}{r}
{\left[\boldsymbol{e}_{1}, \boldsymbol{e}_{1,2}\right] \cup\left[\boldsymbol{e}_{2}, \boldsymbol{e}_{2,3}\right] \cup\left[\boldsymbol{e}_{3}, \boldsymbol{e}_{3,1}\right] \cup \mathcal{C}\left(\left\{\boldsymbol{e}_{1,2}, \boldsymbol{e}_{2,3}, \boldsymbol{e}_{3,1}\right\}\right)} \\
\subseteq \mathcal{R}\left(\left\{\boldsymbol{e}_{1}, \boldsymbol{e}_{2}, \boldsymbol{e}_{3}\right\}\right)
\end{array}
$$

where $\boldsymbol{e}_{1,2}, \boldsymbol{e}_{2,3}, \boldsymbol{e}_{3,1}$ are defined by $\left\{\boldsymbol{e}_{i, j}\right\}=\left[\boldsymbol{e}_{i}, \boldsymbol{e}_{i, i}\right] \cap$ $\left[\boldsymbol{e}_{j}, \boldsymbol{e}_{j, j}\right]$, see Def. 4.

Hence, in this case, an inner bound on the quasiconvex hull is given by the inner triangle and the "arms" of the $\mathrm{T}_{3}$, cf. Figure 2. An outer bound is the convex hull of the three transformation strains.

Next we consider Case 3:

Lemma 3. Let $\boldsymbol{e}_{0}, \boldsymbol{e}_{1}, \boldsymbol{e}_{2} \in \mathcal{S}_{c}^{3 \times 3}$ and $\boldsymbol{e}_{1}, \boldsymbol{e}_{2} \| \boldsymbol{e}_{0}$. Moreover let $\boldsymbol{x} \| \boldsymbol{y} \forall \boldsymbol{x} \in\left(\boldsymbol{e}_{0}, \boldsymbol{e}_{1}\right], \boldsymbol{y} \in\left(\boldsymbol{e}_{0}, \boldsymbol{e}_{2}\right]$ (in particular $\left.\boldsymbol{e}_{1} \| \boldsymbol{e}_{2}\right)$. Then

$$
\mathcal{R}\left(\left\{\boldsymbol{e}_{0}, \boldsymbol{e}_{1}, \boldsymbol{e}_{2}\right\}\right) \supseteq\left[\boldsymbol{e}_{0}, \boldsymbol{e}_{1}\right] \cup\left[\boldsymbol{e}_{0}, \boldsymbol{e}_{2}\right] .
$$

Proof. First we notice that $\mathcal{R}\left(\left\{\boldsymbol{e}_{0}, \boldsymbol{e}_{1}, \boldsymbol{e}_{2}\right\}\right) \supseteq \mathcal{R}\left(\left\{\boldsymbol{e}_{0}, \boldsymbol{e}_{1}\right\}\right) \cup$ $\mathcal{R}\left(\left\{\boldsymbol{e}_{0}, \boldsymbol{e}_{2}\right\}\right)$ and then apply Lemma 1 .

The remaining cases require the following result from [7, Lemma 3.1]:

Lemma 4. Any two-dimensional subspace of $\mathcal{S}_{c}^{3 \times 3}$ either (i) contains precisely one, two or three compatible directions or (ii) is a compatible subspace.

Next we consider Case 4:

Lemma 5. Let $\boldsymbol{e}_{0}, \boldsymbol{e}_{1}, \boldsymbol{e}_{2} \in \mathcal{S}_{c}^{3 \times 3}$ with $\boldsymbol{e}_{1}, \boldsymbol{e}_{2} \| \boldsymbol{e}_{0}$ and $\boldsymbol{e}_{1} \| \mid \boldsymbol{e}_{2}$. Let

$$
\begin{aligned}
& \lambda_{1}:=\max \left\{\lambda \in[0,1) \mid \operatorname{det}\left(\lambda \boldsymbol{e}_{1}+(1-\lambda) \boldsymbol{e}_{0}-\boldsymbol{e}_{2}\right)=0\right\}, \\
& \lambda_{2}:=\max \left\{\lambda \in[0,1) \mid \operatorname{det}\left(\lambda \boldsymbol{e}_{2}+(1-\lambda) \boldsymbol{e}_{0}-\boldsymbol{e}_{1}\right)=0\right\} .
\end{aligned}
$$

(These exist since $\operatorname{det}\left(\boldsymbol{e}_{0}-\boldsymbol{e}_{1}\right)=0$ and $\operatorname{det}\left(\boldsymbol{e}_{0}-\boldsymbol{e}_{2}\right)=0$.) Then $\lambda_{1} \lambda_{2}=0$ and

$$
\mathcal{R}\left(\left\{\boldsymbol{e}_{0}, \boldsymbol{e}_{1}, \boldsymbol{e}_{2}\right\}\right) \supseteq\left\{\begin{array}{c}
\mathcal{C}\left(\left\{\boldsymbol{e}_{0}, \lambda_{1} \boldsymbol{e}_{1}+\left(1-\lambda_{1}\right) \boldsymbol{e}_{0}, \boldsymbol{e}_{2}\right\}\right) \\
\cup\left(\lambda_{1} \boldsymbol{e}_{1}+\left(1-\lambda_{1}\right) \boldsymbol{e}_{0}, \boldsymbol{e}_{1}\right] \\
\mathcal{C}\left(\left\{\boldsymbol{e}_{0}, \boldsymbol{e}_{1}, \lambda_{2} \boldsymbol{e}_{2}+\left(1-\lambda_{2}\right) \boldsymbol{e}_{0}\right\}\right) \\
\cup\left(\lambda_{2} \boldsymbol{e}_{2}+\left(1-\lambda_{2}\right) \boldsymbol{e}_{0}, \boldsymbol{e}_{2}\right]
\end{array} \quad \text { if } \lambda_{2}=0,\right.
$$

Proof. From Lemma 4, we obtain $\lambda_{1} \lambda_{2}=0$ since otherwise we would have four compatible directions and yet $\boldsymbol{e}_{1} \| \boldsymbol{\boldsymbol { e } _ { 2 }}$. We consider the case $\lambda_{2}=0$, the other case is analogous. Let $\boldsymbol{e}_{1}^{\prime}=\lambda_{1} \boldsymbol{e}_{1}+\left(1-\lambda_{1}\right) \boldsymbol{e}_{0}$.

By construction $\boldsymbol{e}_{1}, \boldsymbol{e}_{2} \| \boldsymbol{e}_{1}^{\prime}$ and $\left[\boldsymbol{e}_{1}^{\prime}, \boldsymbol{e}_{1}\right] \not \mid\left[\boldsymbol{e}_{1}^{\prime}, \boldsymbol{e}_{2}\right]$. Thus from Lemma 3, $\mathcal{R}\left(\left\{\boldsymbol{e}_{1}^{\prime}, \boldsymbol{e}_{1}, \boldsymbol{e}_{2}\right\}\right) \supseteq\left[\boldsymbol{e}_{1}^{\prime}, \boldsymbol{e}_{1}\right] \cup\left[\boldsymbol{e}_{1}^{\prime}, \boldsymbol{e}_{2}\right]$. On the other hand, since $\boldsymbol{e}_{0}, \boldsymbol{e}_{1}^{\prime}$ and $\boldsymbol{e}_{2}$ are pairwise compatible, from Lemma $1, \mathcal{R}\left(\left\{\boldsymbol{e}_{0}, \boldsymbol{e}_{1}^{\prime}, \boldsymbol{e}_{2}\right\}\right) \supseteq \mathcal{C}\left(\left\{\boldsymbol{e}_{0}, \boldsymbol{e}_{1}^{\prime}, \boldsymbol{e}_{2}\right\}\right)$.

We conclude that $\mathcal{R}\left(\left\{\boldsymbol{e}_{0}, \boldsymbol{e}_{1}, \boldsymbol{e}_{2}\right\}\right)=\mathcal{R}\left(\left\{\boldsymbol{e}_{0}, \boldsymbol{e}_{1}^{\prime}, \boldsymbol{e}_{2}\right\}\right) \cup$ $\mathcal{R}\left(\left\{\boldsymbol{e}_{1}^{\prime}, \boldsymbol{e}_{1}, \boldsymbol{e}_{2}\right\}\right)$. The result follows.
Finally we address Case 2:

Lemma 6. Let $\boldsymbol{e}_{0}, \boldsymbol{e}_{1}, \boldsymbol{e}_{2} \in \mathcal{S}_{c}^{3 \times 3}$ with $\boldsymbol{e}_{1} \| \mid \boldsymbol{e}_{2}$ and $\boldsymbol{e}_{1}, \boldsymbol{e}_{2}|\not| \boldsymbol{e}_{0}$. Suppose there exist $\lambda_{1}, \lambda_{2}$ such that

$$
\begin{aligned}
& \lambda_{1}=\min \left\{\lambda \in(0,1) \mid \operatorname{det}\left(\lambda \boldsymbol{e}_{1}+(1-\lambda) \boldsymbol{e}_{2}-\boldsymbol{e}_{0}\right)=0\right\}, \\
& \lambda_{2}=\max \left\{\lambda \in(0,1) \mid \operatorname{det}\left(\lambda \boldsymbol{e}_{1}+(1-\lambda) \boldsymbol{e}_{2}-\boldsymbol{e}_{0}\right)=0\right\}
\end{aligned}
$$

(note that we allow for $\lambda_{1}=\lambda_{2}$ ). Then

$$
\begin{aligned}
& \mathcal{R}\left(\left\{\boldsymbol{e}_{0}, \boldsymbol{e}_{1}, \boldsymbol{e}_{2}\right\}\right) \\
& \supseteq \supseteq \mathcal{C}\left(\left\{\boldsymbol{e}_{0}, \lambda_{1} \boldsymbol{e}_{1}+\left(1-\lambda_{1}\right) \boldsymbol{e}_{2}, \lambda_{2} \boldsymbol{e}_{1}+\left(1-\lambda_{2}\right) \boldsymbol{e}_{2}\right\}\right) \cup\left[\boldsymbol{e}_{1}, \boldsymbol{e}_{2}\right] .
\end{aligned}
$$

Otherwise, $\mathcal{R}\left(\left\{\boldsymbol{e}_{0}, \boldsymbol{e}_{1}, \boldsymbol{e}_{2}\right\}\right) \supseteq\left\{\boldsymbol{e}_{0}\right\} \cup\left[\boldsymbol{e}_{1}, \boldsymbol{e}_{2}\right]$.

We omit the proof since it is similar to those for the preceding cases. In the case $\lambda_{1}=\lambda_{2}$ there can be another compatible line.

Lemma 7. Assume that there is $\lambda_{1}, \lambda_{2}$ as in Lemma 6 with $\lambda_{1}=\lambda_{2}$. Then there is at most one $\lambda_{3} \in(0,1)$ solving the equation $\operatorname{det}\left(\lambda_{3}\left(\boldsymbol{e}_{0,0}\right)+\left(1-\lambda_{3}\right) \boldsymbol{e}_{0}-\boldsymbol{e}_{j}\right)=0$ for $j=1$ or $j=2$ with $\boldsymbol{e}_{0,0}:=\lambda_{1} \boldsymbol{e}_{1}+\left(1-\lambda_{1}\right) \boldsymbol{e}_{2}$. If there is such $\lambda_{3}$ then

$$
\begin{aligned}
& \mathcal{R}\left(\left\{\boldsymbol{e}_{0}, \boldsymbol{e}_{1}, \boldsymbol{e}_{2}\right\}\right) \\
& \supseteq \mathcal{C}\left(\left\{\boldsymbol{e}_{j}, \boldsymbol{e}_{0,0}, \lambda_{3} \boldsymbol{e}_{0,0}+\left(1-\lambda_{3}\right) \boldsymbol{e}_{0}\right\}\right) \cup\left[\boldsymbol{e}_{0}, \boldsymbol{e}_{0,0}\right] \cup\left[e_{1}, e_{2}\right] .
\end{aligned}
$$

\section{Lamination upper bound and corresponding numerical approach}

The lamination upper bound relies on earlier works $[9,11$, $12]$ and $[13,14]$. It involves the so-called free energy of mixing $w_{\text {mix }}: \Delta_{n} \rightarrow \mathbb{R}$, which allows to write the quasiconvex envelope of the energy as

$$
Q W(\boldsymbol{e}, \boldsymbol{c})=\sum_{j=1}^{n} c_{j} W_{j}(\boldsymbol{e})+w_{\operatorname{mix}}(\boldsymbol{c}) .
$$

The upper bound of our numerical approach is obtained by an upper bound on the energy of mixing, which satisfies

$$
\begin{aligned}
& w_{\text {mix }}\left(\theta \boldsymbol{c}_{1}+(1-\theta) \boldsymbol{c}_{2}\right) \leq \\
& \theta w_{\text {mix }}\left(\boldsymbol{c}_{1}\right)+(1-\theta) w_{\text {mix }} \boldsymbol{c}_{2}+\theta(1-\theta) \psi\left(\boldsymbol{c}_{1}-\boldsymbol{c}_{2}\right)
\end{aligned}
$$

for all $\boldsymbol{c}_{1}, \boldsymbol{c}_{2} \in \Delta_{n}$, where $\psi$ is given in the references above. In $[13,14]$, an upper bound of $w_{\text {mix }}$ based on (3) was proposed, namely

$$
\begin{aligned}
w_{\text {upp }}(\boldsymbol{c}):=\max \{w(\boldsymbol{c}): & w \text { satisfies (3) and } \\
& \left.w \geq 0 \text { on the vertices of } \Delta_{n}\right\} .
\end{aligned}
$$

Since the condition (3) has to be checked for all $c_{1}, c_{2} \in$ $\Delta_{n}$ with $c_{1} \neq c_{2}$, it is in general not possible to compute $w_{\text {upp }}$. Our approach will be different. Starting from an upper bound of $w_{\text {mix }}$, we will use (3) to obtain a possibly improved upper bound.

Lemma 8. Let $w: \Delta_{n} \rightarrow \mathbb{R} \cup\{+\infty\}$ be an upper bound of $w_{\text {mix }}$, i.e., $w_{\text {mix }}(\boldsymbol{c}) \leq w(\boldsymbol{c})$ for all $\boldsymbol{c} \in \Delta_{n}$. Let $\boldsymbol{c}_{1}, \boldsymbol{c}_{2} \in \Delta_{n}$ be given. Define the function $\tilde{w}: \Delta_{n} \rightarrow \mathbb{R} \cup\{+\infty\}$ as

$\tilde{w}(\boldsymbol{c}):=\min \left\{w(\boldsymbol{c}), \theta w\left(\boldsymbol{c}_{1}\right)+(1-\theta) w\left(\boldsymbol{c}_{2}\right)+\theta(1-\theta) \psi\left(\boldsymbol{c}_{1}-\boldsymbol{c}_{2}\right)\right\}$

if $\boldsymbol{c}=\theta \boldsymbol{c}_{1}+(1-\theta) \boldsymbol{c}_{2}, \theta \in(0,1)$, otherwise set $\tilde{w}(\boldsymbol{c}):=w(\boldsymbol{c})$. Then $\tilde{w}$ is also an upper bound of $w_{\mathrm{mix}}$. 
Note that the construction of $\tilde{w}$ is independent of whether $\boldsymbol{c}_{1}$ and $\boldsymbol{c}_{2}$ are compatible or not.

Proof. Let $\boldsymbol{c}=\theta \boldsymbol{c}_{1}+(1-\theta) \boldsymbol{c}_{2}$ with $\theta \in(0,1)$. It remains to show $\tilde{w}(\boldsymbol{c}) \geq w_{\text {mix }}(\boldsymbol{c})$ in the case $w(\boldsymbol{c}) \geq \theta w\left(\boldsymbol{c}_{1}\right)+(1-$ $\theta) w\left(\boldsymbol{c}_{2}\right)+\theta(1-\theta) \psi\left(\boldsymbol{c}_{1}-\boldsymbol{c}_{2}\right)$. Here, we have

$$
\begin{aligned}
\tilde{w}(\boldsymbol{c}) & =\theta w\left(\boldsymbol{c}_{1}\right)+(1-\theta) w\left(\boldsymbol{c}_{2}\right)+\theta(1-\theta) \psi\left(\boldsymbol{c}_{1}-\boldsymbol{c}_{2}\right) \\
& \geq \theta w_{\text {mix }}\left(\boldsymbol{c}_{1}\right)+(1-\theta) w_{\text {mix }}\left(\boldsymbol{c}_{2}\right)+\theta(1-\theta) \psi\left(\boldsymbol{c}_{1}-\boldsymbol{c}_{2}\right) \\
& \geq w_{\text {mix }}(\boldsymbol{c}),
\end{aligned}
$$

where we used the assumption that $w$ is an upper bound of $w_{\text {mix }}$.

By construction, we have $w_{\text {mix }} \leq \tilde{w} \leq w$, which implies that the idea of Lemma 8 above can be used to obtain iteratively improved upper bounds. To start the iteration, an initial upper bound is needed. Here, it turns out that the trivial Taylor upper bound $w^{0}(\boldsymbol{c})=0$ is enough to get sensible iterates and is exact for the pure variants found at the vertices of the triangle configurations considered in the cases mentioned above.

We present the idea of the method for a mixture of three pure phases as a special case of the twelve possible martensitic variants in NiTi. For convenience we now relabel the strains $1,2,3$. The method can be extended to more phases, but it is expected that the computational cost grows exponentially with the number of phases.

In the simplex $\Delta_{3}$ we generate points with uniform distance $h:=1 / N, N \in \mathbb{N}$ of the form $c=(i / N, j / N,(N-i-$ $j) / N), i=0, \ldots, N, j=0, \ldots, N-i$. Let these points form the set $\boldsymbol{C}$. Our goal is to compute upper bounds of $w_{\text {mix }}$ on all the points $\boldsymbol{c} \in \boldsymbol{C}$.

In the next step, we generate all possible triplets of points $\left(\boldsymbol{c}_{1}, \boldsymbol{c}_{2}, \boldsymbol{c}_{3}\right) \in \boldsymbol{C}^{3}$ with $\boldsymbol{c}_{2}=\frac{1}{2}\left(\boldsymbol{c}_{1}+\boldsymbol{c}_{3}\right)$. Even though alternative construction methods of the triplets, such as the more general $c_{2}=\left(a c_{1}+b c_{3}\right) /(a+b)$, could be used to potentially improve the quality of the bound, they would also increase the computational effort significantly and are therefore not evaluated here. All triplets considered are collected in the set $G$.

Then for any triplet $\left(\boldsymbol{c}_{1}, \boldsymbol{c}_{2}, \boldsymbol{c}_{3}\right) \in G$ we apply (4) to obtain an improved upper bound of $w_{\text {mix }}$. In order to do so, the value of $\psi$ at $\boldsymbol{c}_{1}-\boldsymbol{c}_{3}$ has to be evaluated. Here, we can employ the fact that $\psi$ is positively homogeneous of degree 2. Moreover, the distribution of points of $\boldsymbol{C}$ in the simplex is highly regular and symmetric, and hence the number of evaluations of $\psi$ is much lesser than the number of triplets in $G$. This means that $\psi$ can be evaluated offline, i.e., before the actual iteration starts.

The iteration procedure can be described as follows.

\section{Generate $\boldsymbol{C}, G$.}

2. Evaluate $\psi$ : for all $\left(\boldsymbol{c}_{1}, \boldsymbol{c}_{2}, \boldsymbol{c}_{3}\right) \in G$ compute $\psi\left(\boldsymbol{c}_{1}-\right.$ $\left.c_{3}\right)$.

3. Set initial upper bound: $k=0, w^{0}(\boldsymbol{c}):=0$.
4. Outer iteration, set $w^{k+1}:=w^{k}$.

For each $\left(c_{1}, c_{2}, c_{3}\right) \in G$ : Set

$$
\begin{aligned}
& w^{k+1}\left(\boldsymbol{c}_{2}\right):=\min \left[w^{k+1}\left(\boldsymbol{c}_{2}\right),\right. \\
&\left.\left(w^{k+1}\left(\boldsymbol{c}_{1}\right)+w^{k+1}\left(\boldsymbol{c}_{3}\right)\right) / 2+\psi\left(\boldsymbol{c}_{1}-\boldsymbol{c}_{3}\right) / 4\right]
\end{aligned}
$$

5. Stop if $\left\|w^{k+1}-w^{k}\right\|_{\infty}$ is small enough, otherwise set $k:=k+1$ and return to step 4 .

Due to Lemma 8, all iterates $w^{k}$ generated by the above algorithm are upper bounds of $w_{\text {mix }}$. In our numerical tests, the iteration was stopped if the maximum change between two successive iterates was smaller than $10^{-12}$.

\section{Numerical results}

Next we report about the results of our numerical experiments. The algorithm above was implemented in Matlab and run on a standard desktop computer. The tests were performed with grid size $1 / N=1 / 256$ resulting in $\approx 33,000$ points in $C$. The set $G$ contained $\approx 17.6 \times 10^{6}$ triplets. The minimization contained in the function $\psi$ was evaluated for 240 search directions. We used the cubic-tomonoclinic-I transformation of NiTi, where we took the material parameters summarized in Table 1; these parameters were also used for the upper bounds computed in [8], cf. $\S 5$. The $\mathbb{C}$ tensor is parametrized by three values $\mathbb{C}_{11}=140, \mathbb{C}_{12}=110, \mathbb{C}_{44}=32$. In the figures, the vertices of the triangles are chosen such that the first mentioned strain is in the bottom left corner; the others follow anti-clockwise.

Firstly, we investigated the upper bound for the triple $\left\{\boldsymbol{e}^{(1)}, \boldsymbol{e}^{(6)}, \boldsymbol{e}^{(12)}\right\}$. These transformation strains are pairwise incompatible (Case 1), hence from the above considerations we expect to see $T_{3}$ structures in the upper bound. The total energy surface obtained from the upper bound can be seen in Figure 1, where the vertices $(0,0),(1,0)$, and $\left(\frac{1}{2}, \frac{\sqrt{3}}{2}\right)$ correspond to the phases $\boldsymbol{e}^{(1)}, \boldsymbol{e}^{(6)}$, and $\boldsymbol{e}^{(12)}$. Figure 2 shows the logarithmic scaling of the total energy. Furthermore, the corresponding $\mathrm{T}_{3}$-configuration is superimposed; it shows a very good correspondence.

As can be seen from these figures, the upper bound of the total energy is close to zero in the $T_{3}$ region: the value of the total energy at the barycenter of $\left\{\boldsymbol{e}^{(1)}, \boldsymbol{e}^{(6)}, \boldsymbol{e}^{(12)}\right\}$ is about $1.84 \times 10^{-6}$, while the maximum total energy is of order $3 \cdot 10^{-3}$. This indicates that our approach is successful at computing a sensible upper bound. Moreover, these results prompt the question as to whether the iteratively improved upper bounds would converge to the quasiconvex envelope of the energy if the grid size $1 / N$ goes to zero or if more complex patterns of $G$ are considered.

Secondly, we study Case 2, namely the triple $\boldsymbol{e}^{(1)}, \boldsymbol{e}^{(7)}$, and $\boldsymbol{e}^{(12)}$, cf. Figure 3, as well as the triple $\boldsymbol{e}^{(1)}, \boldsymbol{e}^{(6)}$, and $\boldsymbol{e}^{(8)}$, cf. Figure 4. This also reflects the theoretical behaviour. The first example is related to the last part of Lemma 6: the upper bound is zero at the compatible line $\left[\boldsymbol{e}^{(1)}, \boldsymbol{e}^{(7)}\right]$; otherwise it is clearly not zero, which corresponds to the fact that there are no compatible lines within the triangle. 


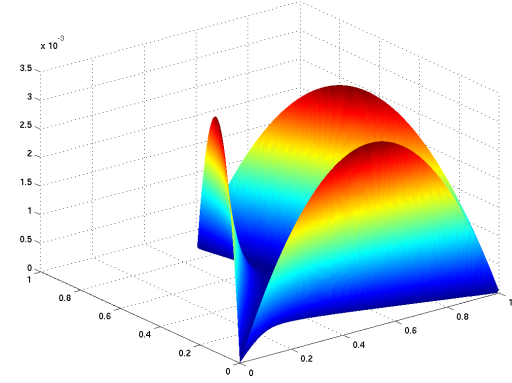

Figure 1. Case 1: Upper bound of the total energy for phases $\left\{\boldsymbol{e}^{(1)}, \boldsymbol{e}^{(6)}, \boldsymbol{e}^{(12)}\right\}$.

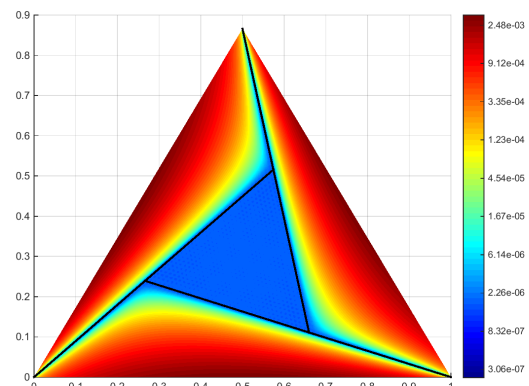

Figure 2. Case 1: Upper bound of the total energy for phases $\left\{\boldsymbol{e}^{(1)}, \boldsymbol{e}^{(6)}, \boldsymbol{e}^{(12)}\right\}$, logarithmic scale. The corresponding $\mathrm{T}_{3}$ configuration is superimposed.

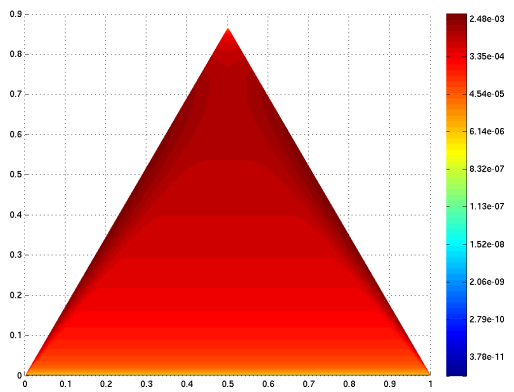

Figure 3. Case 2: Upper bound of the total energy for phases $\left\{\boldsymbol{e}^{(1)}, \boldsymbol{e}^{(7)}, \boldsymbol{e}^{(12)}\right\}$, logarithmic scale. Note that there is a blue line on the bottom of the triangle that reflects the compatibility between the strains $\boldsymbol{e}^{(1)}$ and $\boldsymbol{e}^{(7)}$.

The second example shows exactly the bound asserted in Lemma 7.

Next we present our result for Case 3, in which the strains $\boldsymbol{e}^{(1)}$ and $\boldsymbol{e}^{(7)}$ are compatible as are $\boldsymbol{e}^{(7)}$ and $\boldsymbol{e}^{(10)}$, but $\boldsymbol{e}^{(1)}$ and $\boldsymbol{e}^{(10)}$ are not. Further, there is no other compatible direction in the triangle, i.e., $\lambda_{1}=\lambda_{2}=0$ in Lemma 5 . This computation, see Figure 5, also reflects the corresponding theoretical result, see Lemma 3, which says that an inner bound on the compatible hull is given by the compatible lines $\left[\boldsymbol{e}^{(1)}, \boldsymbol{e}^{(7)}\right]$ and $\left[\boldsymbol{e}^{(7)}, \boldsymbol{e}^{(10)}\right]$.

For Case 4 we show two different results, one for the triple $\left\{\boldsymbol{e}^{(1)}, \boldsymbol{e}^{(2)}, \boldsymbol{e}^{(7)}\right\}$ and one for the triple $\left\{\boldsymbol{e}^{(1)}, \boldsymbol{e}^{(3)}, \boldsymbol{e}^{(12)}\right\}$.

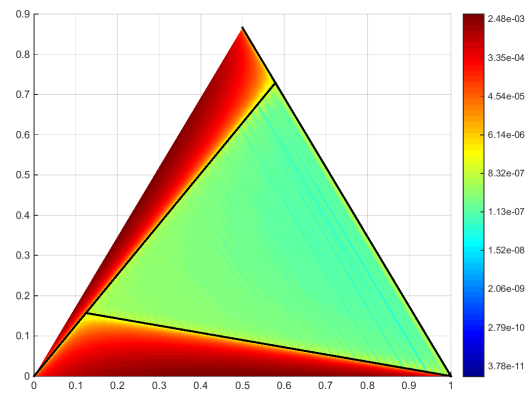

Figure 4. Case 2: Upper bound of the total energy for phases $\left\{\boldsymbol{e}^{(1)}, \boldsymbol{e}^{(6)}, \boldsymbol{e}^{(8)}\right\}$, logarithmic scale. Note that the three compatible lines that occur in this case are superimposed. The numerical values on the right edge of the triangle are in the blue regime.

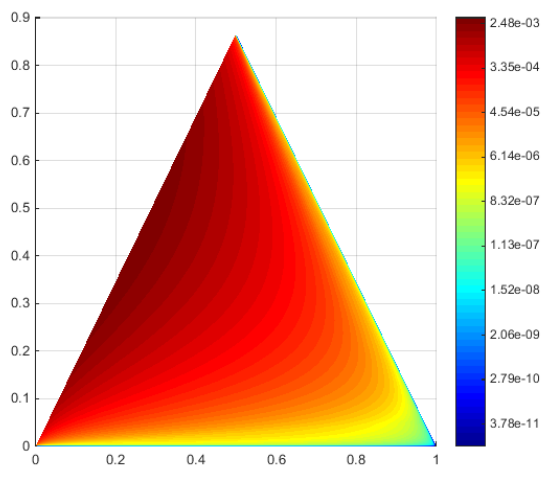

Figure 5. Case 3: Upper bound of the total energy for phases $\left\{\boldsymbol{e}^{(1)}, \boldsymbol{e}^{(7)}, \boldsymbol{e}^{(10)}\right\}$, logarithmic scale.

Both triples have two compatible edges and one incompatible edge as well as an additional compatible line.

First, we present upper bounds of the total energy for the three phases $\left\{\boldsymbol{e}^{(1)}, \boldsymbol{e}^{(2)}, \boldsymbol{e}^{(7)}\right\}$. Here, the phase $\boldsymbol{e}^{(1)}$ is compatible to $\boldsymbol{e}^{(2)}$ and to $\boldsymbol{e}^{(7)}$, while the latter two are incompatible, i.e. we are in Case 4. The upper bound on the total energy by our method can be found in Figure 6 in logarithmic scaling. The value of the total energy at the barycenter of the phases is $1.69 \times 10^{-7}$, while the maximum of the total energy is of the order of $3 \cdot 10^{-3}$. This maximal value yields an estimate of the difference between the upper bound and the convex envelope of the energy. Note that the convex envelope is zero on the whole triangle spanned by the three strains considered. Hence, as in [8], we obtain that the difference between these two hulls is small.

As can be observed, the upper bound closely approximates the expected set of zeros of the total energy, which is sketched by a solid line in the figure. Thus it confirms the non-symmetric shape of the inner bound on the quasiconvex hull. Moreover, the logarithmic figure appears to indicate that there is a sharp transition between the zero set of the energy and energy states outside of the inner triangle.

Second, we studied $\left\{\boldsymbol{e}^{(1)}, \boldsymbol{e}^{(3)}, \boldsymbol{e}^{(12)}\right\}$. This again shows a non-symmetric triangle, which however is much smaller, cf. Figure 7. Note the correspondence with the theoreti- 


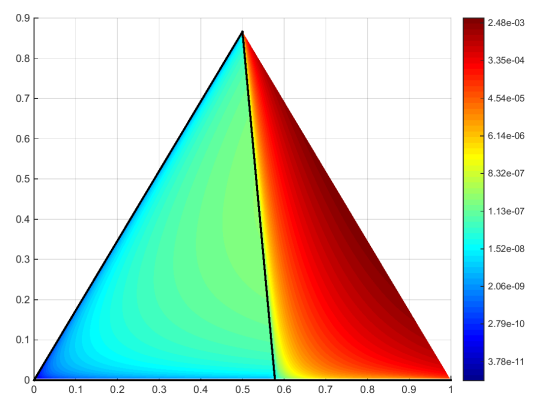

Figure 6. Case 4: Upper bound of the total energy for phases $\left\{\boldsymbol{e}^{(1)}, \boldsymbol{e}^{(2)}, \boldsymbol{e}^{(7)}\right\}$, logarithmic scale. Note that the compatible lines are superimposed.

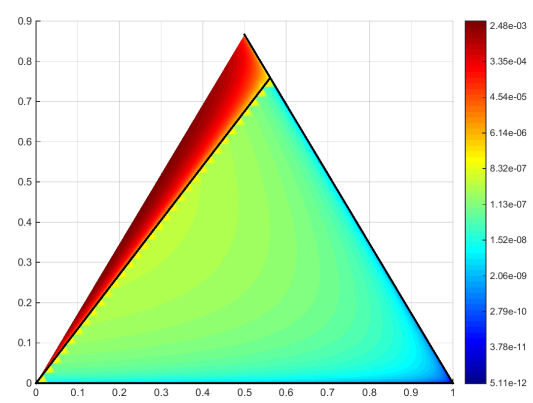

Figure 7. Case 4: Upper bound of the total energy for phases $\left\{\boldsymbol{e}^{(1)}, \boldsymbol{e}^{(3)}, \boldsymbol{e}^{(12)}\right\}$, logarithmic scale. Note that the compatible lines are superimposed.

cal inner bound on the set of recoverable strains, which is again indicated by a solid line in the figure.

Finally, our computations show that the lamination upper bound in Case 5 of the three pairwise compatible strains $\left\{\boldsymbol{e}^{(1)}, \boldsymbol{e}^{(2)}, \boldsymbol{e}^{(3)}\right\}$ is almost zero on the whole triangle (no figure shown). Since in this case, the lamination convex hull equals the convex hull, this is exactly as expected.

\section{Conclusion}

In this article we have demonstrated a close relationship between theoretical predictions on inner bounds of compatible hulls of transformation strains and numerical upper bounds on the quasiconvex envelopes of the corresponding energy. This work was motived by observations in [8] about the lamination upper bound which yields energy values showing some relation to the corresponding $\mathrm{T}_{3}$-configurations. With the new algorithm presented in this article we obtain much better correspondence. Indeed, the algorithm shows that the lamination upper bound is (almost) zero at the $\mathrm{T}_{3}$-configurations.

Similarly, for the other cases of triples of strains we obtain a strikingly correspondence between the inner bound on the quasiconvex hull of the strains and the (almost) zero set of the upper bound of the quasiconvex envelope of the energy.

Another observation made in [8] is related to the convex envelope. In the case of pairwise compatible triples of strains, the convex envelope happens to be identically zero on the triangle spanned by the three strains. It turns out that the relative difference between the energy values of the lamination upper bound and the convex envelope is small, even in the setting of $\mathrm{T}_{3} \mathrm{~s}$, which involves incompatibilities, the difference is $3 \cdot 10^{-3}$. On the other hand it shows well that there is an interesting structure that can be seen in the lamination upper bound on the energy as well as in the inner bound on the set of recoverable strains. Whether these structures are also present in the quasiconvex envelopes and hulls remains an open problem.

\section{References}

[1] J.M. Ball, R.D. James, Archive for Rational Mechanics and Analysis 100, 13 (1987)

[2] K. Bhattacharya, Microstructure of martensite: Why it forms and how it gives rise to the shape-memory effect, Oxford series on materials modelling (Oxford University Press, 2003),

[3] S. Müller, in Calculus of variations and geometric evolution problems, edited by F. Bethuel, G. Huisken, S. Müller, K. Steffen (Springer, 1999), pp. 85-210

[4] B. Dacorogna, Direct methods in the calculus of variations, Applied mathematical sciences, 2nd edn. (Springer, 2007)

[5] K. Bhattacharya, Continuum Mech. Thermodyn. 5, 205 (1993)

[6] K. Bhattacharya, R.V. Kohn, Archive for Rational Mechanics and Analysis 139, 99 (1997)

[7] I.V. Chenchiah, A. Schlömerkemper, Archive for Rational Mechanics and Analysis 207, 39 (2013)

[8] R. Fechte-Heinen, A. Schlömerkemper (preprint)

[9] R.V. Kohn, Continuum Mech. Thermodyn. 3, 193 (1991)

[10] K. Bhattacharya, N.B. Firoozye, R.D. James, R.V. Kohn, Proc. Roy. Soc. Edinburgh A 124, 843 (1994)

[11] M. Avellaneda, G.W. Milton, Bounds on the effective elasticity tensor of composites based on two-point correlations, in Proceedings of the ASME EnergyTechnology Conference and Exposition, edited by D. Hui, T. Koszic (New York, 1989)

[12] L. Tartar, Proc. Roy. Soc. Edinburgh A 115, 193 (1990)

[13] S. Govindjee, A. Mielke, G.J. Hall, Journal of the Mechanics and Physics of Solids 51, I (2003)

[14] A. Mielke, in Multifield Problems: State of the Art, edited by A.M. Sändig, W. Schiehlen, W.L. Wendland (Springer, 2000), pp. 96-103 